\title{
Cloudlet Vision: Examined Cloudlets Performance while End-Users use Multimedia Contents
}

\author{
Abdullah Saleh AI-Saleh ${ }^{1,2^{*}}$ and Shaya Abdullah Al-Shaya ${ }^{2}$ \\ 'Department of Information Engineeting, Florence University, Florence, Italy; aalsaleh@unifi.it \\ 2Department of Computer Science, Majmaah University, Al Majmaah - 15341, Saudi Arabia; shaya@mu.edu.sa
}

\begin{abstract}
Objective: Mobile devices have been found to perform high-performance processing tasks, as smart phones are increasingly becoming popular accompanied by development of mobile applications. Battery life, memory, weight, and heat dissipation limit the computational resources of the device. Cloud computing can be one of the best solutions to overcome mobile devices limitations. However, some of the problems that can be experienced in cloud computing include costly roaming charges and long-latency of cellular radio access. The purpose of this research paper is to develop an approach, which uses coordination between cloudlets, to not only enhance the performance of cloud access, but also bring new experience among mobile device users. Methods: A research review methodology was adopted to undertake analysis of the existing experimental studies on cloudlet computing to understand their proposed models, protocols, and theoretical framework for enhancing access and security of data from remote cloud using mobile devices, where cloudlets serve as intermediary. Location-Aware Service Deployment (LASD) algorithm was proposed to enhance cloudlet coordination through reduction of latency. Findings: The findings of existing experimental studies reveal several conclusive observations. There are benefits provided by the use of cloudlets over the remote cloud. They include faster processing of an application, faster data transfer as well as reducing the use of mobile resources. In the study, we have examined the data latency in the process of connections and data transfer between cloudlets including the distance cloud servers with and without coordination, using multimedia files. Application: Coordinated cloudlets will help clients to have their multimedia files to be transferred faster within the environment of cloud-computing.
\end{abstract}

Keywords: Cloud Computing, Cloudlet, Data, Multimedia

\section{Introduction}

Mobile computing is an unstoppable wave, especially with the rapid advancements in the ICT and commerce. Mobile gadgets, such as smartphone and tablet PC, are increasingly enhancing human life in terms of communication because of their effectiveness and convenience. By accessing various services through mobile apps like the iPhone and Google apps, mobile users are living with rich experiences. These mobile applications are installed on mobile devices and access respective services from remote servers through the internet or localized wireless networks. However, mobile technology is facing two types of challenges: resources regarding storage, bandwidth, battery life, memory, and, weight; and communications regarding security and mobility. The improvement of service qualities is attributed to delay due to these resources limitations significantly. Cloud technology plays an important role in exchanging data among various types of users. It enables the users to share network resources that ultimately provide self-assured solutions and providing reliable ways of supporting the improvements of network and communication technologies. However, alongside the development of mobile apps and the increasing adoption of long-latency, cloud computing and costly roaming charges of cellular radio access, the risks of possible concerns have emerged. Moreover, a noisy neighbor can negatively affect other users' cloud access. The noisy neighbor

*Author for correspondence 
effect causes other virtual machines and applications, which share the infrastructure to cause uneven cloud network performance. Cloud computing is the future of the infrastructure of computing. Amazon, Google, as well as Salesforce are some example of cloud service providers. They offer users services such as infrastructure, platform, and software as services in a cost effective way.

Customers use SaaS to access and use software or application programs online from the cloud, for example, Google Spreadsheet and Google Docs. Through IaaS, customers can access servers and networks and use the cloud storage to save files. Amazon provides elastic cloud storage where computing has turned into a real reality, where each computing layer is the stack to provide stretchable and infinite storage capacity. On the other hand, PaaS provides access to operating systems and middle services. Consequently, mobile applications operate without necessarily requiring significant management efforts or interactions of the service providers.

Cloud computing technology uses a network of many computers, which help in providing cloud services to different users. Some cloud services include access to the database for running websites, file storages, and launching software applications. These are common services that users can access from a single computer or a computer server. It is impossible to isolate one single computer as a separate device providing the services in the cloud. The entire network is responsible for the servicing. In cloud computing, such heavy lifting functions like storing files, as well as running software are provided by the network of computers. The user's computer only runs interphase software applications to enable them to interact with the cloud. E-mail is a good example of this aspect. Users may use a web browser for their e-mail. They use emails to receive, read, delete, write, and send messages. However, the actual e-mail software and the files are not on their computer. They are located in the cloud.

The mobile cloud computing idea is a basic cloud computing feature. Increasingly, the idea has attracted entrepreneurs as the mobile technology niche is an emerging market. By introducing new experiences and IT solutions at lower costs, the development of mobile applications is revolutionized by mobile cloud computing. Integrating cloud computing technology into the mobile environment is the basic idea of mobile cloud computing, and the integration is needed to support new mobile appli- cations with different services being accessed from the cloud. With this integration, mobile users can enjoy more services, which were never thought as possible, through their mobile devices by taking full advantage of cloud computing technology.At its simplest definition, mobile cloud computing can be described as an infrastructure that aids mobile users to access, process and store data away from their mobile phones ${ }^{1}$. Mobile devices provide just user interface, but all the computing power and storage are cloud-based. To have mobile applications not relying on the computing power of mobile device chipset will be a paradigm shift, as centralized computing platforms would access from the cloud. With the advent of mobile cloud computing, it will be possible for the mobile devices to use simple browsers to access the centralized applications for personalized service from the cloud with a high probability of and success rate, access, and speed of task execution, in a more secure environment.

\section{Virtualization of Cloudlets Infrastructure}

Two terms are frequently confused, but they are very different. They are Cloud computing and virtualization. Cloud is based on virtualization, but the latter on its own does not constitute a cloud even when infrastructure is completely virtualized. Cloudlets are small-scale mobile data centers that provide cloud services using mobile devices including smartphones, tablets, and wearables. A cloudlet is a product of integrating cloud computing into a mobile computing environment ${ }^{2}$. It is at the center of the 3-layer hierarchy: Device-Cloudlet-Cloud. Satyanarayanan viewed the cloudlet concept as a trusted, resource-rich computing closer to mobile users through a wireless access point ${ }^{3}$. Although a limited view of endto-end latency initially drove cloudlets, its role in mobile computing has been critical especially in hostile environments. A cloudlet surrogates the real cloud and acts as a doorway between the cloud and the mobile device ${ }^{4}$. In addition, cloudlet is described as a small-scale cloud closer to mobile users, who access it through LAN networks ${ }^{5}$. The past authors have included discussion of face recognition technology in their works, recommending the use of the architecture of MObile Cloud-based Hybrid Architecture (MOCHA) and its early performance results ${ }^{6}$ (Figure 1). 


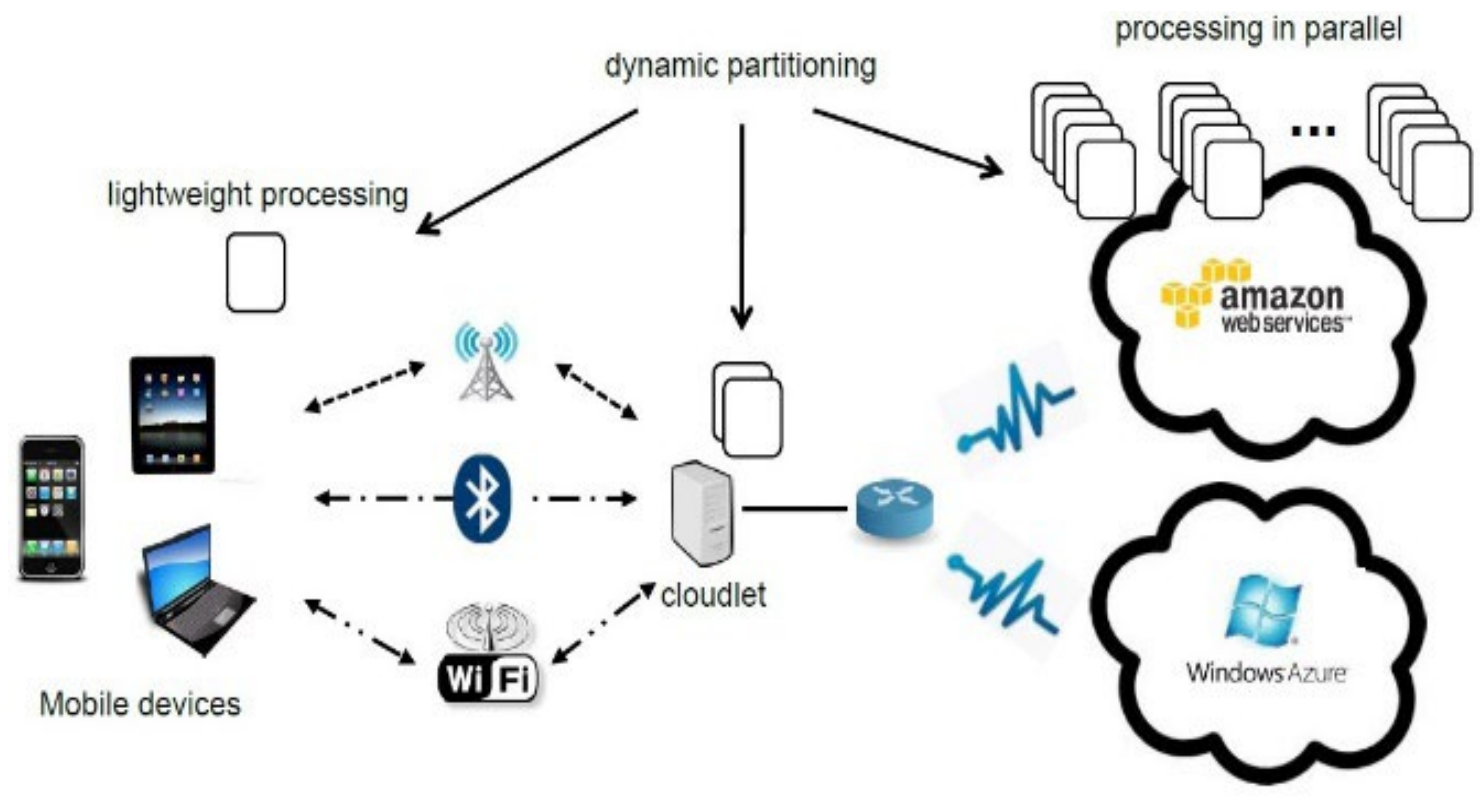

Figure 1. The MOCHA architecture.

Using a cloudlet can simplify the complexity involved in achieving the bandwidth demand from various users during interactions through request and reception of media products like high definition videos and high-res images ${ }^{7}$. Moreover, cloudlet has some benefits; such as reduced equipment cost, so one can avoid several costly access points used to improve network coverage ${ }^{8}$. It is attained as the wireless termination points are kept as light as possible. Furthermore, MOCHA decreases access latency because it brings certain applications and network functions near the mobile users. Simple and flexible network manager, such as a cloudlet, tends to be self-managed requiring little or no professional interventions, minimizing OPEX and CAPEX by eliminating cost ineffective middle-boxes, along with their management hurdles. The management complications may arise due to the network functions through virtualized technology and the likelihood of emergence of personalized services based on certain geographical locations. Additionally, provisioning can be carried out remotely using the device without necessarily installing software in accessing services.

Despite extensive research in the fields of cloudlet and cloud computing, there is a lack of studies showing the importance of coordination between these cloudlets. Therefore, the action aimed to carry out research on cloud computing and cloudlets to determine the merits and demerits of existing methods and proposed methodolo- gies for improvement and to develop concepts and techniques of cloudlets coordination environment (managing these cloudlets for better security and performance).

\section{Research Methodology}

Over time, the number of accessing the internet by mobile phones has steadily increased. Mobile hardware is resource-poor characterized by many drawbacks as compared to client hardware and static server. Factors such as heat dissipation, storage memory, battery strength, and weight limit the computational resources of the mobile device. In mobile technology, cloud computing definitely offers solutions to these limitations of the mobile hardware. With cloudlets, instantiating tailored virtual machines from the cloud and running the relevant software in a thin client browser is possible for mobile users. Several studies have examined the cloudlet in an in-depth manner for example Ceselli and his team showed an understanding view of this new paradigm of cloud computing. They examined the design problem of the cloudlet networks. The model proposed by these researchers show that there's an association between virtual machines and mobile clients and they are allotted to cloudlets. The design of cloudlet network process starts by determining the installation location for cloud facilities, then allocating access point sets like base stations, while allowing migrations of virtual machines in tandem with 
data mobility based on a service-level agreement. There are two modes of migration: live and bulk migration. These modes are expressed as functions of cloud service requirements, where bulk migration is given higher preference for delay-strict services like support for augmented reality. Live migration is preferable for applications with less strict delay requirements. The model by Ceselli and his team aimed at doing the following:

- Finding an optimal network design and installing and allocating base stations to their corresponding cloudlets, and

- Finding an optimal traffic route to the cloudlets, despite the fact that the main objective is to deliver the best design policies instead of the operational planning.

The model provided an elaborate framework for designing cloudlet network for metropolitan area mobile access networks. The researcher also described the design problem, as well as a planning mode for the mobility of the virtual machine using both live and bulk migrations. They also undertook an extensive comparison of different planning alternatives for situations involving real datasets from a cellular network. Characteristics being compared include the number of active cloudlets, performance for cloud services and the volume of traffic migrated.

In addition, the researchers also reviewed high gain, deducing it using the perspective of the mobility of virtual machine and users in the network. They found appropriate cloud services and planning theories, in the wide view of traffic volume migrated through cloudlet network.

Jemaa, Pujolle and Pariente presented a new architectural design for WLAN networks managed by carriers, which leveraged virtualization concepts of the network function ${ }^{8}$. They used two virtual machines that ran Xubuntu OS and FreeBSD OS. They tested the delay performance in three cases:

1. Delay occurring between the video server and user's device,

2. Round trip time measurement for both proposed and referenced designs between the gateway and the user's device, and

3. Delay occurring between the user's device and video server.

They concluded that WLAN cloudlet solution has several benefits, including agility, flexibility, and costs. They also showed a cloudlet-based WLAN architecture. Manzalini and his team addressed the potential effect of disruptive technologies and solutions on the development of carrier's network ${ }^{10}$. They identified some of the key technical challenges in migrations, dynamic allocations, and virtual machine arrangement ensembles across interconnected peripheral networks. The hosting infrastructure Virtual Box is implemented by two multi-core servers running with a Linus Cent OS as the VM hypervisors. The VM hypervisors used in the experiment are virtual single-core boxes. One serves as the access router and the other serves as the video server. The access router provides a connection between the user and the server hint ensembles across interconnected peripheral networks. Migration latency can be kept at minimal by dimensioning two VMs were with the minimum memory capacity preferably $512 \mathrm{MB}$ for both of them and disk with a storage capacity of at least 7.2 GB and 1.3 GB allocated to the video server and access router respectively, required to run functions. A gateway for live movement of the total network infrastructure is provided by the VM teleporting function of the Virtual Box. The two hosting servers which emulate two remote data centers are connected to the user interfaces by an Ad hoc link. The researchers concluded that the future networking infrastructures shall be constituted by thousands of virtual resources pertinent to computation, storage network input/output controlled in a dynamic fashion based on customers' demand, business objectives, and changing situations.

The advancement in standard hardware and the emergent of disruptive technologies in cloud computing, including NFV and SDN, is inevitable. Introduced the idea of cloudlet properties; like a lifetime, reachable time, and size, and examined the mobile cloudlet domain in mobile cloud computing by studying the computing performance of cloudlets and their properties ${ }^{11}$. They demonstrated that the number of nodes and frequency of meeting in the network dictates the resource-rich units an initiator can be connected while taking part in computing services. Additionally, if a task is tolerant to delay, the discontinuous connection has the insignificant negative impact on a mobile cloudlet performance. These researchers also came up with lower and upper bounds of computing ability of mobile cloudlets, including speed and bandwidth. The initiator can utility these limits to make decisions on whether to either upload a task to mobile cloudlets or remote clouds. The researchers have proposed future areas of research particularly on 
the design and incorporation of mobile applications into mobile cloudlets while investigating their viability and performance.

In proposed security implementations in a new cloudlet design ${ }^{12}$, to enhance security, they designed a new cloudlet mesh for establishing trusted cloudlet computing by identifying authentication sequence, together with encryption protocols for secured communications from clouds, cloudlet servers, and mobile devices, and vice versa. With this design, these researchers aimed at improving two aspects.

The first one is hierarchy-based security design where there is an established trust chain between cloud platforms, cloudlet, and mobile devices. The second aspect that requires improvement involves processing of security analytics that are predictive in nature at the backend cloud for blocking viruses and malware. Focusing on realtime blockage of malicious programs or codes, or high level of agility to intrusion may help in safeguarding the cloud's integrity. The researcher also suggested the adoption of multiple cloudlets to increase network coverage to support numerous mobile devises as their cloudlets supported Wi-Fi wireless networks. They also used multiple cloudlets to enhance coordination between them making it possible to construct an alert and shield system for all connected mobile devices. Multiple cloudlets also enabled load balancing and cashing in offloading computational tasks from the remote cloud. They demonstrated these benefits by comparing three cloudlets in a composite mesh to the use of just one cloudlet in detecting intrusions. Even at a false positive rate of 0.006, composite mess performs between 10 and $20 \%$, which is higher than that of single intrusion detective system.

In presented a large-scale Body Area Networks (BANs) system with respect to data collection from cloudlet-based. Their aim was to reduced costs associated with the end-to-end packet by using a cloudlet-based system to collect data dynamically from the cloud ${ }^{13}$.

They also aimed at reducing overall delay involved in the end-to-end packet through a dynamic choice of a neighboring cloudlet. The execution of Cloud Simulator tool $^{14}$ is as in Teachcloud ${ }^{15}$, for the actualization of cloudlet-based Body Area Networks model. As they implement their model, every virtual machine was allocated to the available hardware resources, such as $\mathrm{CPU}(\mathrm{s})$, which provide an environment for multi-core technology. More significantly, cloudlet system had adequate memory capacity for each physical server. The storage capacity was mod- erate with a potential of reaching TB scale. The cloudlet had several transceivers for the reception and transmission of the data packet to as well as from the Body Area Network users and these supported major functionalities. The researchers used a cloudlet unit that used a standard architecture of $\mathrm{x} 86$, Xen as VM monitor, VM allocation to host with minimum usage as an allocation policy, a TB storage, 8GB memory, two 4-core CPUs, space-shared VM scheduler, $10 \mathrm{Mbps}$ bandwidth, and communication tools involving $15 \mathrm{~km}$ ranged WiMAX and $100 \mathrm{~m}$ ranged Wi-Fi. They used two primary parameters to gauge the performance of cloudlet-based Body Area Networks regarding data collection: packet transmission parameter and packet delay parameter. Packet delay and packet transmissions measure the communication energy and delay expenditure between the enterprise cloud or cloudlet and PDA device, respectively. These researchers concluded that increased number of VCs minimizes delay and communication energy because of wider coverage of Wi-Fi network.

Clinch and his team explored the location of application affects the cloudlet users' experience ${ }^{16}$. They wondered whether the location should be at a distant cloud possessing a characteristically high network latency or closer to the user. They found it necessary to quantify the latency effect on the cloudlet user's experience. By emulating mobile device on a Mac Mini with Ubuntu operating system connected wireless network, they simplified their packet tracing and automation. However, it was noted that they gauged task performance using a real mobile device rather than emulator in their experimental research. While deploying three physical cloudlet machines, four cloud virtual machine instances, and seven servers, they connected to the cloudlet with opensource VNC client. Cloud VM instances provided access to the Amazon elastic cloud. This experiment was done in the United States and the European Union. They found that there was a significant difference between the two regions indicating a longer latency with a significant effect on gameplay, a tested Users' experience. These outcomes helped in design and location choices. Through this experiment, it has been found that application location has a potential impact on the users' experience and performance of applications being sourced from the cloudlet or remote cloud.

Described several challenges inherent to mobile cloud computing including how they affected the distribution system design. The study also reviewed the main 
outcomes Coda and Odyssey systems and explained the future opportunities on five areas can be exploited into mobile cloud computing. These areas include semantic validators and callbacks, caching parameters, adaptation analysis, resource revocation, and conversion of location observations into global estimations ${ }^{17}$.

In proposed a concept of cloudlet that involves the management of applications at a component level, rather than transferring the entire virtue environment to cloudlets from the remote cloud. They also demonstrated the competencies and benefits of using real-time augmented reality from a mobile application perspective ${ }^{18}$.

In proposed community clinic solution that is embedded somewhere between the mobile users and cloud, in the cloudlet, with the goal of decreasing costs of deploying many datacenters in the cloud and save the energy being used by the mobile devices ${ }^{19}$. While determining the effect of mobility, Li and Wang conducted experimental research to determine the probability of cloud access, the speed of task execution, and success rates ${ }^{20}$. They discovered that the probability of cloudlet access is the ratio of average inter-connection time to average connection time between the connected mobile device and the cloudlet. They also discovered that the success rate and execution speed of a task does not only depend on the speed of cloudlet computation and computation demand of that task, but also the probability of access in the cloudlet.

In proposed a protocol for data security for cloudletbase architecture ${ }^{21}$. Using the characteristics of an ideal forward secrecy, they integrated base station into cloudlet.
Their protocol for data security prevented any unauthorized user from accessing data. It also prevented data exposure to the cloud service provider. The proposed protocol for data security contains several elements: cloud, cloudlet, and data owner (mobile device user). The data owner uploaded encrypted files to the cloud, share it, and access it regardless of location and time of the day. Their protocol model used cloudlets as intermediate between the cloud and mobile devices, where each cloudlet served 100 mobile devices. These experimental researchers have several assumptions: 1 . cloudlets can be trusted, where each maintains often accessed file cache, 2 . the data owner populates the list of data sharers, 3. cloud servers have elastic storage capacity and sufficient computing power, 4 . Cloud servers remained online throughout, 5. Every file has a unique ID, and 6. Before any upload, a new session is given its key. The protocol eliminated tasks requiring intensive computation and allowed simple wireless gadgets to store and retrieve data from the cloud. From the security analysis, the proposed protocol prevent illegal access to the stored data, thus the integrity of the mobile cloud computing is maintained.

\section{Software Design}

The purpose of this project was developed to facilitate the communication process between servers and clients in the cloud through Cloudlets. Cloudlets serve as intercessors between clients and remote cloud servers. Clean separation has been enabled through the use of virtual machine

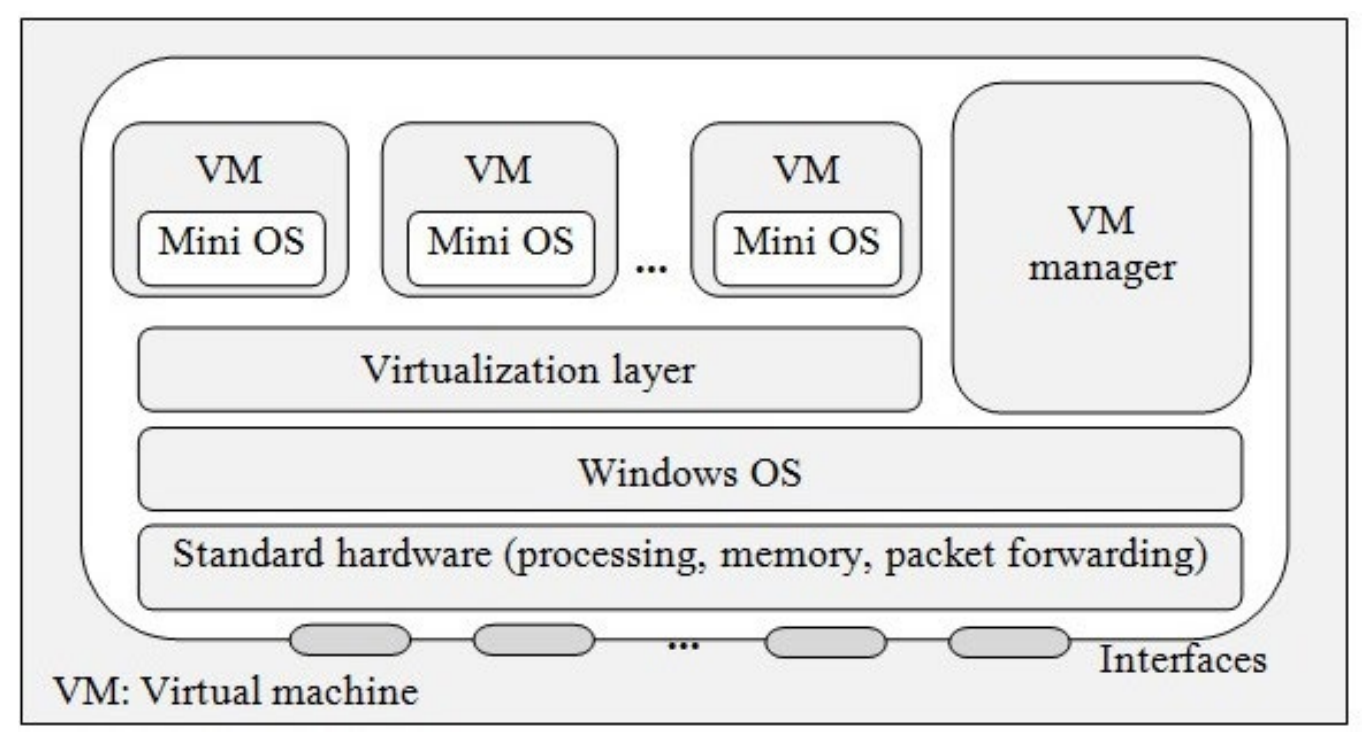

Figure 2. Generalized experiment architecture. 


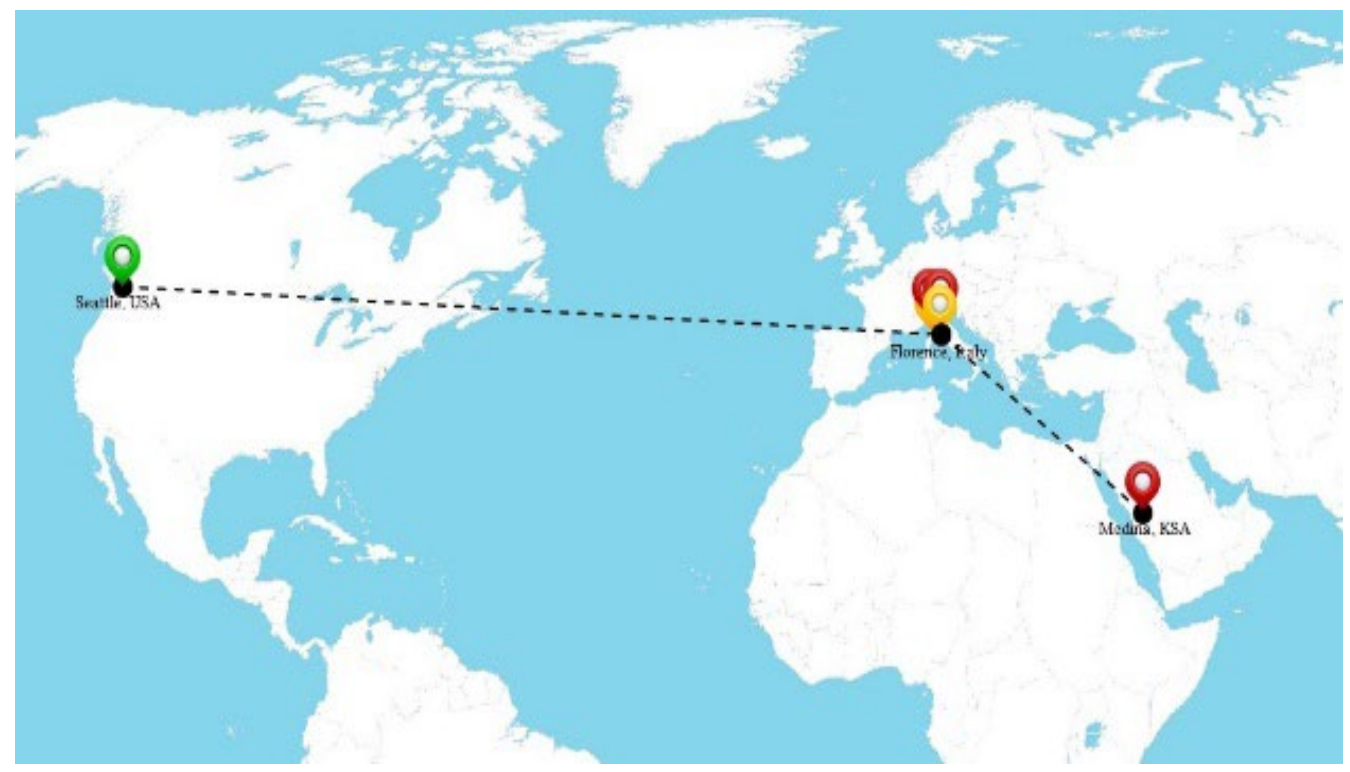

Figure 3. The Geographic distribution of the experiment.

cloud machines. The complex problem of the software configuration in cloudlet can be simplified by transferring a preconfigured machine to the floating cloud. A transient guest software environment can be clearly encapsulated and separated from the permanent host in the cloudlet. The stable host-to-guest interface helps increase compatibility between Cloudlet and mobile devices. Figures 2-3 show the generalized experiment architecture and the geographic distribution of the experiment.

The study set up 7 virtual machines: $1 \mathrm{VM}$ server in the cloud, 3 cloud cloudlets and $3 \mathrm{VM}$ clients in the cloud via a public network between the United States, UU, Saudi Arabia, and Italy.

The cloud's remote VM server has been installed in Seattle, USA. UU. Its IP address is (67.161.106.95). It had the following characteristics (Intel Core i5, 20 GB of the disk, $2.67 \mathrm{GHz}$, and $1 \mathrm{~GB}$ of RAM) and running VMware Workstation 9.0 and Windows Web Server 2008. The three VM cloudlets were all installed in Florence, Italy, and all have a shared IP address (79.52.120.186). They had the following features (Intel Core i3-4005U, 10 GB disk, $1.70 \mathrm{GHz}$, and $512 \mathrm{RAM}$ ) and were running VMware Workstation 9.0 and Windows Web Server 2008.

The three VM customers were installed in Italy and Saudi Arabia as follows: with an IP address (95.187.39.3), the first client was installed in Medina, Saudi Arabia. It had the features of 5 GB of disk, 512 RAM and 2.93 $\mathrm{GHz}$ Intel Core 2 Duo processor, and ran VMware Workstation 9.0 and Windows 7. With an IP address
(188.217.84.118), the second customer was installed in Florence, Italy. It had the characteristics of (Intel Core i5-3317U CPU, 5GB disk, $1.20 \mathrm{GHz}$ and $512 \mathrm{RAM}$ ) and running VMware Workstation 9.0 and Windows 7. The third customer was set up in Florence, Italy, and has the IP address (79.52.120.186). Its characteristics are (Intel Core i3-4005U, 5 GB disk, $1.70 \mathrm{GHz}$, and $512 \mathrm{RAM}$ ) and running VMware Workstation 9.0 and Windows 7. Table 1 summarizes the used hardware in the experiment.

Table 1. Hardware used for the experiment

\begin{tabular}{|l|l|l|l|l|}
\hline $\begin{array}{l}\text { Computer } \\
\text { No. }\end{array}$ & CPU & Disk & RAM & Speed \\
\hline Comp. 1 & Intel Core i5 & $20 \mathrm{~GB}$ & $1 \mathrm{~GB}$ & $2.67 \mathrm{GHz}$ \\
\hline Comp. 2 & $\begin{array}{l}\text { Intel Core i3- } \\
4005 \mathrm{U}\end{array}$ & $10 \mathrm{~GB}$ & $512 \mathrm{MB}$ & $1.70 \mathrm{GHz}$ \\
\hline Comp. 3 & $\begin{array}{l}\text { Intel Core i3- } \\
4005 \mathrm{U}\end{array}$ & $10 \mathrm{~GB}$ & $512 \mathrm{MB}$ & $1.70 \mathrm{GHz}$ \\
\hline Comp. 4 & $\begin{array}{l}\text { Intel Core i3- } \\
4005 \mathrm{U}\end{array}$ & $10 \mathrm{~GB}$ & $512 \mathrm{MB}$ & $1.70 \mathrm{GHz}$ \\
\hline Comp. 5 & Intel Core 2 Duo & $5 \mathrm{~GB}$ & $512 \mathrm{MB}$ & $2.93 \mathrm{GHz}$ \\
\hline Comp. 6 & $\begin{array}{l}\text { Intel Core i5- } \\
3317 \mathrm{U}\end{array}$ & $5 \mathrm{~GB}$ & $512 \mathrm{MB}$ & $1.70 \mathrm{GHz}$ \\
\hline Comp. 7 & $\begin{array}{l}\text { Intel Core i3- } \\
4005 \mathrm{U}\end{array}$ & $5 \mathrm{~GB}$ & $512 \mathrm{MB}$ & $1.70 \mathrm{GHz}$ \\
\hline
\end{tabular}

The study also compared two data exchange scenarios: the direct connection between a remote cloud and a cloudlet, 
and the multiple connections between the remote cloud and cloudlets. Therefore, the project's main purpose is the measurement of the data's latency in two separate scenarios in cloudlets environments.

The project's internal structure is as follows: the data will be stored on the remote server in the cloud and based on customer requests, it will be requested by cloudlets. The implementation of the clients and servers will be done with JAVA.

\section{Implementation Details}

The project provides in-depth analysis for a better understanding of how it works. Therefore, this section provides a general description of the key details. The remote server in the cloud started with a specific machine in a specific port. In the end, three clouds start. The remote cloud server's address and port are known to each cloudlet. In coordination mode, the addresses and port of others are known to the point clouds. Meanwhile, the addresses and ports of the others are not known by point clouds in noncoordination mode.

During the implementation, a cloud coordination algorithm was proposed: Algorithm Location- Aware Service Deployment (LASD). The algorithm contains two execution phases. The first phase involves the application of K-means dividing mobile devices into serialized clusters based on geographic locations and then implementing a service instance for each cluster on a remote server in the cloud. Determining if the deployment configuration of the service performed in the first phase satisfies the latency constraints of a network by the action of the prediction, is the goal of the second phase of the LASD. The algorithm is configured to iterate between the two phases by multiplying the service instances already used, regardless of the number of iterations, until reaching the latency restriction of a network or after reaching the maximum service instances allowed by resource or provider administrators of services.

The idea of K-means ${ }^{22}$, which can be traced in 1967 by MacQueen, is a clustering algorithm that redistributes data in an iterative manner to a unit of clusters for the purpose minimizing value in Eq. 1.

$$
\sum_{i=1}^{k} \sum_{x \in s_{i}}\left(x-c_{i}\right)^{2}
$$

This clustering algorithm formulation, $\mathrm{k}=$ clusters, $\mathrm{x}=$ data from data set, $=$ the center of cluster . In meeting the goal of cloudlet coordination, the formulated algorithm performs iteration or loop in the two phases until maximal service instances are achieved. The first phase of the algorithm involves distribution of each data particular () to a unit data set based on Eq. 2 .

$$
S_{i}=\left\{x_{p}:\left\|x_{p}-c_{i}\right\|^{2} \leq\left\|x_{p}-c_{j}\right\|^{2} \forall_{j}, i \leq j \leq k\right\}
$$

When is closest to then, is distributed to . Second phase involves updating the center of instantaneous data set as indicated by Eq. 3 .

$$
c_{i}=\frac{1}{\left|S_{i}\right|} \sum_{x \in s_{i}} x
$$

In the second phase, contains $\left|S_{i}\right|$ as the quantity of data items. When the update phase is complete, the data sets are emptied, and iteration continues.

In the project, two scenarios were used to test the speed: first using direct connections among the cloudletsand the remote cloud server; the second is using cloud connection coordination before the connecting the remote cloud server. Each client or server runs in its own folder containing mp3 and mp4 files. Each client downloads files from the cloudlet. If the cloudlet does not have this file, it will request that it be downloaded from the remote cloud server or other cloudlets depending on the scenario being run.

SCENARIO 1: client 1 requests the file 1 . If the cloudlet 1 has this file, it sends the file 1 to the client 1 . Otherwise, the cloudlet 1 communicates the remote server in the cloud. Then, the remote server sends this file to cloudlet 1 and cloudlet 1 forward it to client 1 . Figure 4 shows the cloudlet architecture in scenario 1.

SCENARIO 2: Client 1 requests file 1 . If cloudlet 1 has this file, it sends the file to the client 1 . Otherwise, cloudlet 1 question cloudlet 2 and if cloudlet 2 has this file, it sends it to cloudlet 1 and cloudlet 1 sends it to client 1 . If the 2 cloudlet does not have this file, cloudlet 1 question other cloudlets. If all cloudlets do not have this file, cloudlet 1 asks the cloud remote server to download this file, then cloudlet 1 sends it to client 1 . Figure 5 shows the cloudlet architecture in scenario 2. 


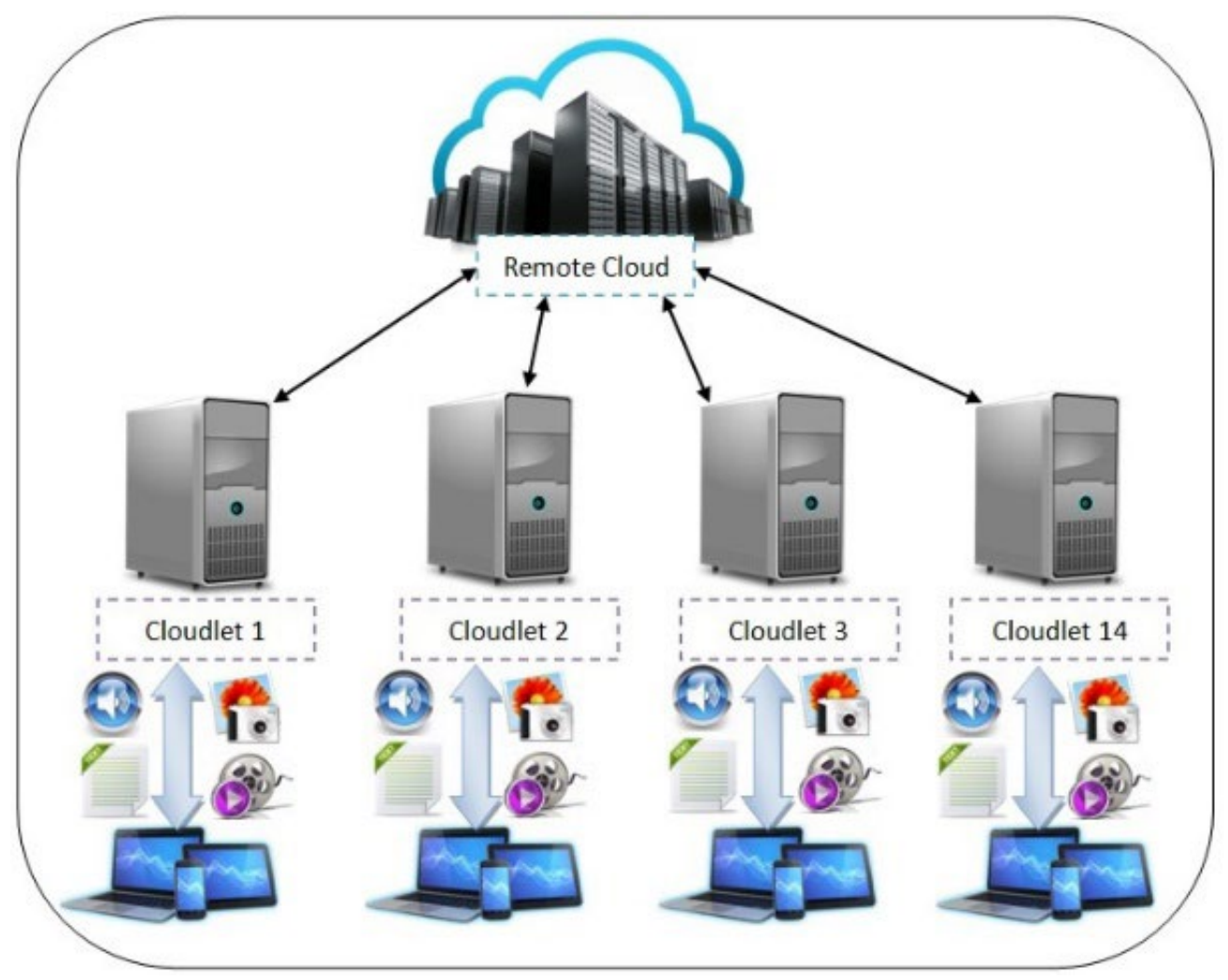

Figure 4. Cloudlet architecture in scenario 1.

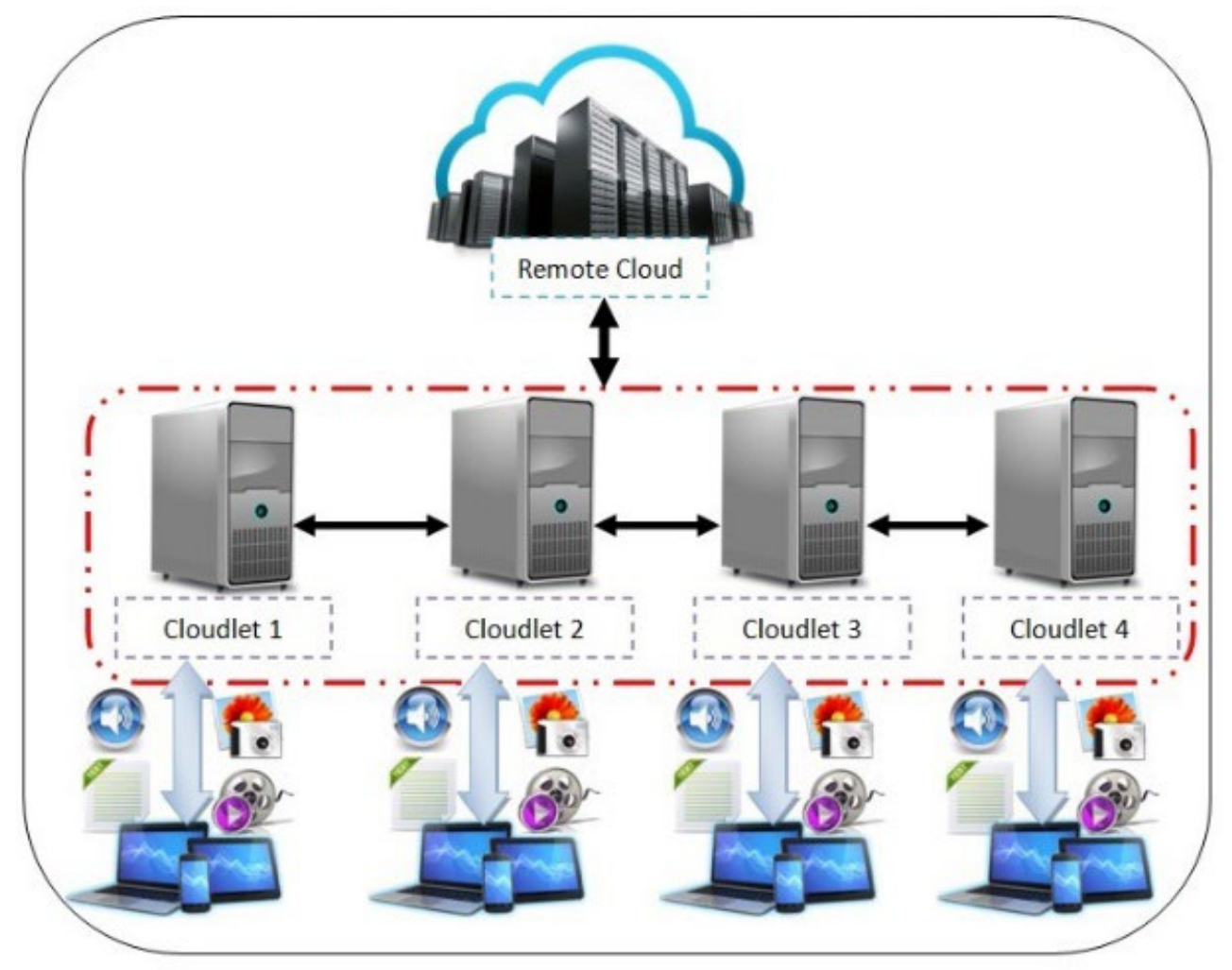

Figure 5. Cloudlet architecture in scenario 2. 


\section{Experimental Results}

The experimental results were collated from 7 virtual machines; 1 as a remote server in the cloud, 3 as cloudlets and 3 as clients. The study took measurements from 3 clients in two scenarios of multimedia data exchange: the first direct connections among cloudlets and remote server were used and the second coordinated connections between cloudlets and remote server were used. In addition, latency was measured during the exchange of multimedia data content between the server in the cloud and three clients, and the delay was recorded. Cloudlets were added to the cloud server and three clients, and the new latency was measured and recorded. Figure 6, it is clear that adding cloudlets would improve the latency. It took less time to send data to the three clients when they did their requests. In addition, the performance of the three clients increased when the cloudlets coordinated with each other. It is concluded that the three clients obtained the requested data in the second scenario, where they requested coordinated cloudlets, faster than in the first scenario where they requested cloudlets without coordination.

Based on results showed in Table 2, performance improvements were observed when implementing the cloudlet architecture. The results showed that the performance improvements would be between (7-44\%); while multimedia files are used for data exchange. In addition, performance can be even better when we use the coordinated Cloudlets architecture. The results showed that the improvements were between 1 and 27\% compared to cloudlets that do not coordinate with each other.

To summarize the two scenarios examined in the cloud-based architecture, we can say that through a

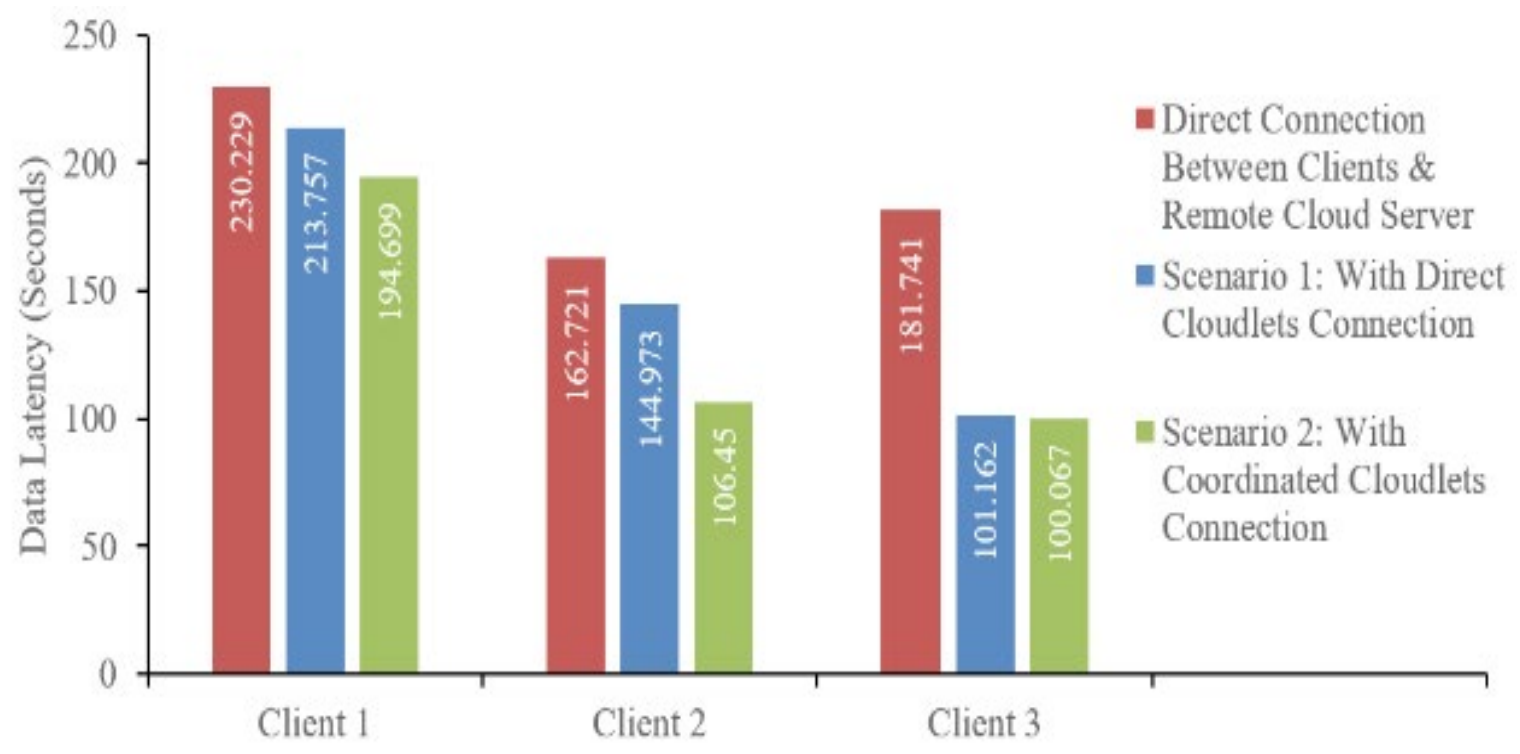

Figure 6. Performance comparison.

Table 2. Summary of results

\begin{tabular}{|l|l|l|l|}
\hline Items & $\begin{array}{l}\text { Time needed to get data with direct } \\
\text { connection between clients and remote } \\
\text { cloud without cloudlets in between }\end{array}$ & $\begin{array}{l}\text { Time needed to get data in } \\
\text { scenario 1 (Cloudlets not } \\
\text { coordinating with each other) }\end{array}$ & $\begin{array}{l}\text { Time needed to get data } \\
\text { in scenario 2 (Cloudlets } \\
\text { coordinating with each other) }\end{array}$ \\
\hline $\begin{array}{l}\text { Client } 1 \\
\text { (IP: } 95.187 .39 .3)\end{array}$ & 230229 milliseconds & $\begin{array}{l}213757 \text { milliseconds } \\
\approx-7 \%\end{array}$ & $\begin{array}{l}194699 \text { milliseconds } \\
\approx-9 \%\end{array}$ \\
\hline $\begin{array}{l}\text { Client } 2 \\
\text { (IP: } 188.217 .84 .118)\end{array}$ & 162721 milliseconds & $\begin{array}{l}144973 \text { milliseconds } \\
\approx-11 \%\end{array}$ & $\begin{array}{l}106450 \text { milliseconds } \\
\approx-27 \%\end{array}$ \\
\hline $\begin{array}{l}\text { Client } 3 \\
\text { (IP: 79.52.120.186) }\end{array}$ & 181741 milliseconds & $\begin{array}{l}101162 \text { milliseconds } \\
\approx-44 \%\end{array}$ & $\begin{array}{l}100067 \text { milliseconds } \\
\approx-1 \%\end{array}$ \\
\hline
\end{tabular}




\section{- \% Needed to Complete Data Exchange Between Clients \& Cloud Server \\ n \% Needed to Complete Data Exchange Using Cloudlets \\ - \% Needed to Complete Data Exchange Using Coordinated Cloudlets}

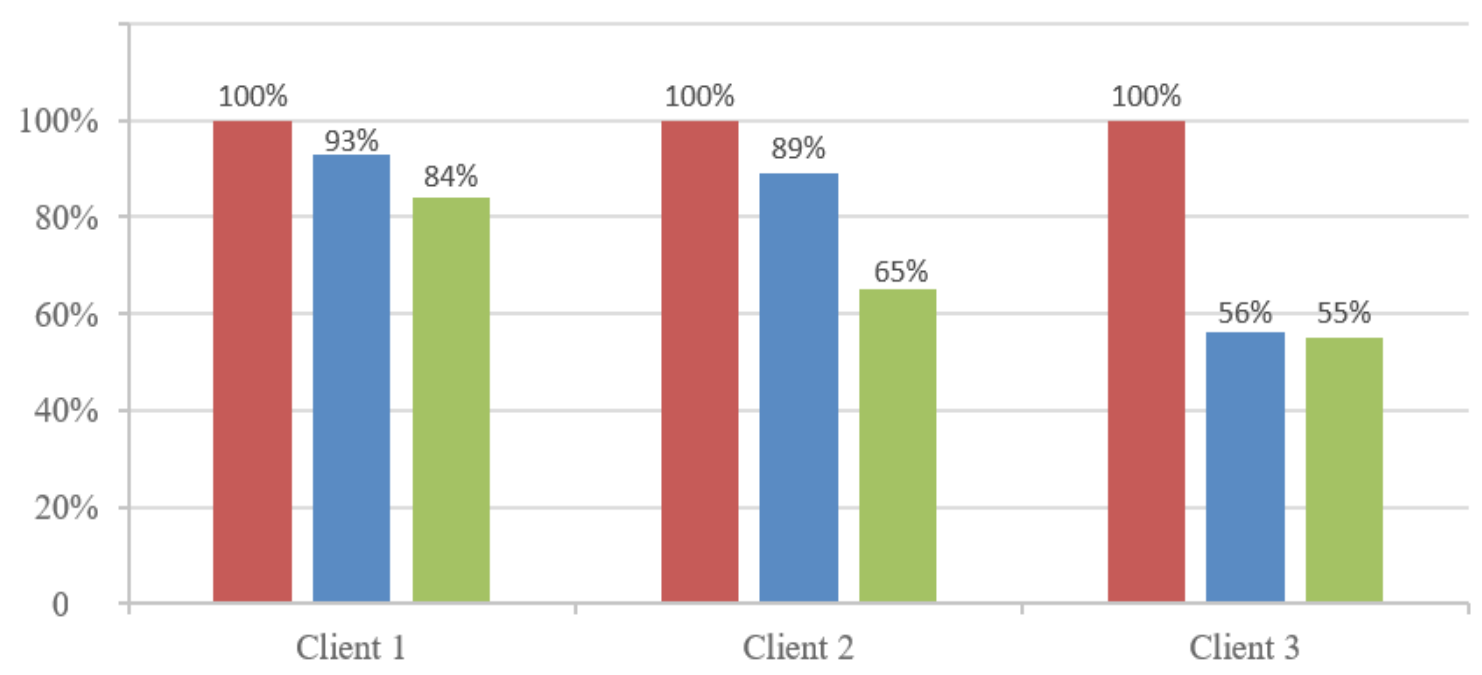

Figure 7. Performance improvement.

cloudlet, the first model directly connects the distance cloud with the clients; the second model connects users with the distance cloud after connecting other clouds for faster and better communication. Therefore, cloudlet coordination significantly supports the cloud computing infrastructure environment by enabling faster and more reliable connections.

Latency has been converted to percentages for a better illustration of the effect of using cloudlet architecture and coordinated cloudlet architecture for transmission times. In Figure 7, it was found that adding cloudlets that coordinate with each other would only use $84 \%, 65 \%$ and $55 \%$ of the time required to transmit data between the three clients and the remote server in the cloud comparing to the three clients directly connecting the remote server.

\section{Conclusion and Future Work}

The study has proposed a coordination method based on cloudlet-based architecture. The proposed solution tested the speed of data in cloudlet environment, with and without coordination, with multimedia type of data. Coordination analysis showed that proposed method could transfer the data faster than the normal cloudlet architecture, using the proposed cloudlet coordination algorithm. The simulation outcomes showed that the pro- posed LASD algorithm effectively minimizes not only the latency constraint of a network, but also service instances. The lower the number of service instances is the lower the latency constraint of a network. Service deployment minimizes instances of data overload in the cloud servers. The benefit of cloudlets in the area of internet of things shall be examined further in the future, especially in designing of high-level service deployment through the proposed LASD algorithm. The algorithm shall be important in accessing cloud services involving HD video streaming.

\section{References}

1. Kitanov S, Davcev D. Mobile Cloud Computing Environment as a Support for Mobile Learning. The Third International Conference on Cloud Computing, GRIDs, and Virtualization; 2012. p. 1-7. PMid: 22077203

2. Jaiswal AS, Thakare VM, Sherekar SS. Performance-based analysis of cloudlet architectures in mobile cloud computing, International Journal of Computer Applications. International Journal of Computer Applications, National Conference on Recent Trends in Information Security (NCRTIS); 2015. p. 1-7.

3. Satyanarayanan M. Mobile computing: the next decade. The 1st ACM Workshop on Mobile Cloud Computing and Services: Social Networks and Beyond (MCS); 2010. p. 1-6. crossref. 
4. Satyanarayanan M, Lewis G, Morris E, Simanta S, Boleng J, Ha K. The Role of Cloudlets in Hostile Environments. IEEE Conference on Pervasive Computing; 2013. p. 40-49. crossref, crossref.

5. Bahtovski A, Gusev M. Cloudlet Challenges. 24th DAAAM International Symposium on Intelligent Manufacturing and Automation; 2013.

6. Soyata T, Muraleedharan R, Funai C, Kwon M, Heinzelman W. Cloud-vision: real-time face recognition using a mobileclouldlet-cloud acceleration architecture, IEEE. 2012; 1-8.

7. Satyanarayanan M, Bahl P, Caceres R, Davies N. The case for VM-based cloudlets in mobile computing, IEEE Pervasive Computing. 2009; 8(4): p. 14-23. crossref.

8. Jemaa F, Pujolle G, Pariente M. Cloudlet- and NFV-based carrier Wi-Fi architecture for a wider range of services, Annals of Telecommunications. 2016; 71(11-12):617-24. crossref.

9. Ceselli A, Premoli M, Secci S. Cloudlet Network Design Optimization. IFIP Networking Conference (IFIP Networking); 2015. p. 1-9. crossref.

10. Manzalini A, Minerva R, Callegati F, Cerroni W, Campi A. Clouds of virtual machines in edge networks, IEEE Communication Magazine. 2013; 51(7):63-69. crossref.

11. Li Y, Wang W. The unheralded power of cloudlet computing in the vicinity of mobile devices, Wireless Networking Symposium.Proceedings of IEEE Global Telecommunications Conference; 2013. p. 4994-99. PMid: 23857602 PMCid:PMC3778106.

12. Shi Y, Abhilash S, Hwang K. Cloudlet Mesh for Securing Mobile Clouds from Intrusions and Network Attacks. IEEE 3rd International Conference on Mobile Cloud Computing. Services and Engineering; 2015. p. 109 - 118. crossref.

13. Quwaider M, Jararweh Y. Cloudlet-based for Big Data Collection in Body Area Networks. IEEE 8th International Conference for Internet Technology and Secured Transactions; 2013. p. 137-41. crossref, crossref.
14. Calheiros RN, Ranjan R, Beloglazov A, De Rose CA, Buyya R. Cloud Sim: A toolkit for modeling and simulation of cloud computing environments and evaluation of resource provisioning algorithms, Software-Practice and Experience. 2011; 44(1):23-50. crossref.

15. Jararweh Y, Alshara Z, Jarrah M, Kharbutli M, Alsaleh M. Teachcloud: A cloud computing educational toolkit. In: Proceedings of the 1st International IBM Cloud Academy Conference (ICA CON 2012). IBM, Research Triangle Park, NC, USA; 2012.

16. Clinch S, Harkes J, Friday A, Davies N, Stayanarayanan M. How Close is Close Enough? Understanding the Role of Cloudlets in Supporting Display Appropriation by Mobile Users. IEEE International Conference on Pervasive Computing and Communications; 2012. p. 122-27. crossref.

17. Satyanarayanan M. Fundamental challenges in mobile computing,. Proceedings of the Fifteenth Annual ACM Symposium on Principles of Distributed Computing. 1996; 1-7. crossref.

18. Verbelen T, Simoens P, De Turck F, Dhoedt B. Cloudlets: Bringing the cloud to the mobile user, Proceedings of the third ACM workshop on mobile cloud computing and services. 2012; 29-36. crossref.

19. Zhang J, Xiong T, Lou W. Community Clinic: Economizing Mobile Cloud Service Cost via Cloudlet Group. IEEE 11th International Conference on Mobile Ad Hoc and Sensor Systems; 2014. p. 208-16.

20. Li Y, Wang W. Can Mobile Cloudlet Support Mobile Application? IEEE Conference on Computer Communications; 2014. p. 1060-68.

21. Jindal M, Dave M. Data Security Protocol for Cloudlet Based Architecture. IEEE International Conference on Recent Advances and Innovations in Engineering; 2014. crossref.

22. MacQueen J. Some methods for classification and analysis of multivariate observations. In: Proceedings of the Berkeley Symposium on Mathematical Statistics and Probability; 1967. p. 281-97. PMCid: PMC1748987. 